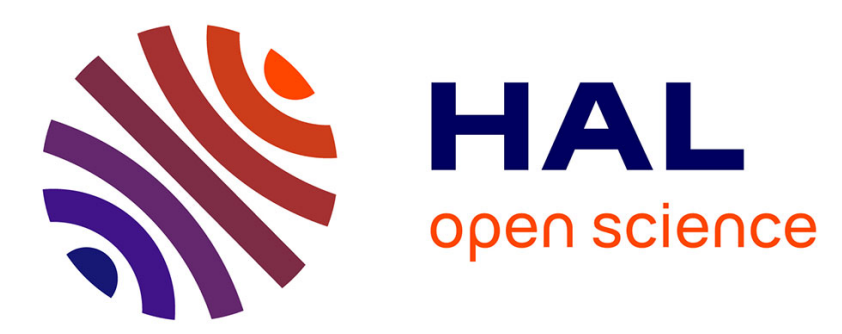

\title{
Effects of Repeated-Sprint Training in Hypoxia on Sea-Level Performance: A Meta-Analysis
} Franck Brocherie, Olivier Girard, Raphael Faiss, Grégoire P Millet

\section{To cite this version:}

Franck Brocherie, Olivier Girard, Raphael Faiss, Grégoire P Millet. Effects of Repeated-Sprint Training in Hypoxia on Sea-Level Performance: A Meta-Analysis. Sports Medicine, 2017, 47 (8), pp.16511660. 10.1007/s40279-017-0685-3 . hal-02545023

\section{HAL Id: hal-02545023 https://hal-insep.archives-ouvertes.fr/hal-02545023}

Submitted on 16 Apr 2020

HAL is a multi-disciplinary open access archive for the deposit and dissemination of scientific research documents, whether they are published or not. The documents may come from teaching and research institutions in France or abroad, or from public or private research centers.
L'archive ouverte pluridisciplinaire HAL, est destinée au dépôt et à la diffusion de documents scientifiques de niveau recherche, publiés ou non, émanant des établissements d'enseignement et de recherche français ou étrangers, des laboratoires publics ou privés. 


\section{Sports Medicine \\ Effects of repeated-sprint training in hypoxia on sea-level performance: a meta- analysis \\ --Manuscript Draft--}

\begin{tabular}{|c|c|}
\hline Manuscript Number: & SPOA-D-16-00350R2 \\
\hline Full Title: & $\begin{array}{l}\text { Effects of repeated-sprint training in hypoxia on sea-level performance: a meta- } \\
\text { analysis }\end{array}$ \\
\hline Article Type: & Systematic Review \\
\hline \multicolumn{2}{|l|}{ Funding Information: } \\
\hline Abstract: & 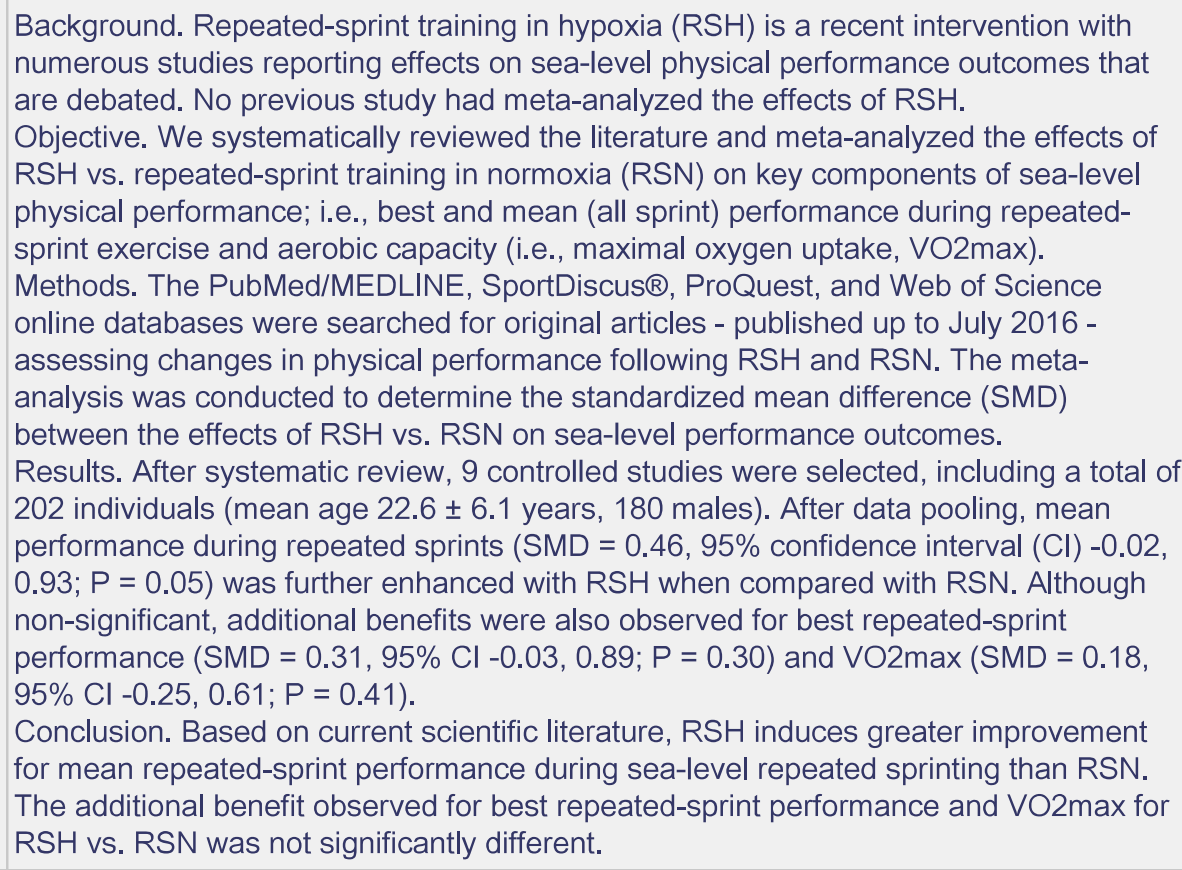 \\
\hline Corresponding Author: & $\begin{array}{l}\text { Franck Brocherie, PhD } \\
\text { ISSUL, Institute of Sports Sciences, University of Lausanne, Switzerland } \\
\text { Lausanne, SWITZERLAND }\end{array}$ \\
\hline \multicolumn{2}{|l|}{$\begin{array}{l}\text { Corresponding Author Secondary } \\
\text { Information: }\end{array}$} \\
\hline Corresponding Author's Institution: & ISSUL, Institute of Sports Sciences, University of Lausanne, Switzerland \\
\hline \multicolumn{2}{|l|}{$\begin{array}{l}\text { Corresponding Author's Secondary } \\
\text { Institution: }\end{array}$} \\
\hline First Author: & Franck Brocherie, PhD \\
\hline \multicolumn{2}{|l|}{ First Author Secondary Information: } \\
\hline \multirow[t]{4}{*}{ Order of Authors: } & Franck Brocherie, $\mathrm{PhD}$ \\
\hline & Olivier Girard, PhD \\
\hline & Raphael Faiss, PhD \\
\hline & Gregoire P Millet, PhD \\
\hline \multicolumn{2}{|c|}{ Order of Authors Secondary Information: } \\
\hline Author Comments: & $\begin{array}{l}\text { Dear Editor-in-Chief, } \\
\text { We thank you for your remarks regarding our revised manuscript "Effects of repeated- } \\
\text { sprint training in hypoxia on sea-level performance: a meta-analysis". We have revised } \\
\text { our manuscript according to your recommendations and your editorial suggestion that }\end{array}$ \\
\hline
\end{tabular}


Title: Effects of repeated-sprint training in hypoxia on sea-level performance: a metaanalysis

Running title: Meta-analysis of repeated-sprint training in hypoxia

\title{
Authors:
}

Franck Brocherie $^{1,2}$, Olivier Girard ${ }^{1,3}$, Raphaël Faiss ${ }^{1}$, Grégoire P. Millet ${ }^{1}$

\begin{abstract}
Affiliations:
${ }^{1}$ ISSUL, Institute of Sports Sciences, University of Lausanne, Switzerland,

${ }^{2}$ Laboratory Sport, Expertise and Performance (EA 7370), Research Department, French Institute of Sport (INSEP), Paris, France.

${ }^{3}$ Aspetar Orthopaedic and Sports Medicine Hospital, Athlete Health and Performance Research Centre, Doha, Qatar.
\end{abstract}

\section{Corresponding author:}

Franck Brocherie

ISSUL, Building Geopolis, Campus Dorigny, University of Lausanne, CH-1015, Lausanne, Switzerland.

Ph. +41216923294

Fax. +41216923293

Email franck.brocherie@unil.ch

Abstract: 250 words; Key points: 136 words

Text-only count (introduction through discussion): 3098 words

Number of tables: 2

Number of figures: 4 


\begin{abstract}
Background. Repeated-sprint training in hypoxia (RSH) is a recent intervention with numerous studies reporting effects on sea-level physical performance outcomes that are debated. No previous study had meta-analyzed the effects of RSH.
\end{abstract}

Objective. We systematically reviewed the literature and meta-analyzed the effects of RSH vs. repeated-sprint training in normoxia (RSN) on key components of sea-level physical performance; i.e., best and mean (all sprint) performance during repeated-sprint exercise and aerobic capacity (i.e., maximal oxygen uptake, $\mathrm{VO}_{2} \mathrm{max}$ ).

Methods. The PubMed/MEDLINE, SportDiscus ${ }^{\circledR}$, ProQuest, and Web of Science online databases were searched for original articles - published up to July 2016 - assessing changes in physical performance following RSH and RSN. The meta-analysis was conducted to determine the standardized mean difference (SMD) between the effects of RSH vs. RSN on sea-level performance outcomes.

Results. After systematic review, 9 controlled studies were selected, including a total of 202 individuals (mean age $22.6 \pm 6.1$ years, 180 males). After data pooling, mean performance during repeated sprints $(\mathrm{SMD}=0.46,95 \%$ confidence interval $(\mathrm{CI})-0.02,0.93 ; \mathrm{P}=0.05)$ was further enhanced with RSH when compared with RSN. Although non-significant, additional benefits were also observed for best repeated-sprint performance $(\mathrm{SMD}=0.31,95 \% \mathrm{CI}-0.03,0.89 ; \mathrm{P}=$ $0.30)$ and $\mathrm{VO}_{2} \max (\mathrm{SMD}=0.18,95 \% \mathrm{CI}-0.25,0.61 ; \mathrm{P}=0.41)$.

Conclusion. Based on current scientific literature, RSH induces greater improvement for mean repeated-sprint performance during sea-level repeated sprinting than RSN. The additional benefit 
observed for best repeated-sprint performance and $\mathrm{VO}_{2} \max$ for $\mathrm{RSH}$ vs. $\mathrm{RSN}$ was not significantly different. 


\section{Key points}

- Repeated-sprint training in hypoxia (RSH) is a recent hypoxic training method aimed at improving physical performance. Its effectiveness on repeated-sprint ability is clear when compared with control (i.e., no repeated-sprint training) but is debated when compared with repeated-sprint training in normoxia (RSN).

- This meta-analytic review shows that RSH in reference to RSN is more efficient to significantly improve mean repeated-sprint performance while an additional positive (but non-significant) effect on best repeated-sprint and maximal oxygen uptake $\left(\mathrm{VO}_{2} \mathrm{max}\right)$ is reported.

- $\mathrm{RSH}$ requires sport-specific adjustment of the main training variables including length/duration of sprint and recovery intervals, exercise:recovery ratio, inter-set recovery duration and/or session frequency. Further investigations manipulating these variables are needed to improve RSH prescription and shed more light on the postulated underlying mechanisms (i.e., compensatory vasodilatation, micro-vascular oxygen delivery (fasttwitch fibers) and specific skeletal muscle molecular adaptations). 


\section{Introduction}

In elite sport, the difference in performance between athletes is tiny [1]. In order to gain a competitive edge, the majority of elite endurance athletes such as distance runners or road cyclists are regularly training in altitude/hypoxia via different available strategies [2-4]. The traditional panorama of hypoxic/altitude training [2] has recently been updated $[3,5]$ to reflect the development of innovative hypoxic interventions currently used by team- and or racquet-sport athletes [6]. The implementation of these methods has been facilitated by technological advances and development of a new generation of hypoxic devices (e.g., normobaric hypoxic chamber, nitrogen-enriching or $\mathrm{O}_{2}$-filtering portable devices, mobile inflatable hypoxic marquees) [7].

Nowadays, ‘live low-train high' (LLTH) methods are increasingly popular. In particular the socalled 'repeated-sprint training in hypoxia' (RSH) [8], which is based on the repetition of 'allout' efforts of short $(\leq 30 \mathrm{~s})$ duration interspersed with short incomplete recoveries, is acquiring unprecedented attractiveness. This model differs from the traditional 'intermittent hypoxic training' since exercise intensity is maximal, thereby allowing a high fast-twitch fibers recruitment [8-12]. In 2013, when compared to similar repeated-sprint training in normoxia (RSN), the pioneer RSH study demonstrated larger maximal repeated sprinting performance improvement and fatigue resistance in normoxia [9]. With a quite low 'hypoxic dose', RSH is unlikely to stimulate the erythropoietic pathway $[13,14]$. Rather its efficacy relies on specific skeletal muscle tissue adaptations mediated by an oxygen-sensing pathway (i.e., hypoxic inducible factors) [15-18], likely to be fiber-type specific [8].

Although a recent systematic review [19] has discussed the efficacy of LLTH to enhance sealevel physical performance, the effectiveness of RSH is passionately debated $[20,21]$ with 
critics' main concerns relating to fatigue criteria definition and/or repeated-sprint test control [22, 23]. However, the growing interest for implementing RSH in different sports at an elite or professional level (e.g., Roland Garros Tennis Academy, Welsh national rugby team, Swedish National Wintersport Centre, French alpine and cross-country ski national teams) highlights the question of the effectiveness of RSH and therefore underlines the importance of a meta-analytic review of RSH. Therefore, we systematically reviewed and meta-analyzed the effects of RSH on best and mean performance during repeated sprinting and aerobic capacity (i.e., maximal oxygen uptake, $\left.\mathrm{VO}_{2} \max \right)$.

\section{Methods}

\subsection{Literature Search}

The review and analysis was conducted in accordance with PRISMA (Preferred Reporting Items for Systematic Reviews and Meta-analyses) statement guidelines [24]. A systematic search of the research literature was conducted for randomized controlled trials studying the effects of RSH interventions on sea-level physical performance. The search included articles published up to July 2016 using PubMed/MEDLINE, SportDiscus ${ }^{\circledR}$, ProQuest, and Web of Science online databases. The following terms were searched for in 'all fields' - [(hypoxi* OR normobar* OR altitude) AND (repeated sprint train* OR high-intensity intermittent train*) while the terms (patients OR obes*) were excluded (using NOT). Analysis was restricted to 'English language' and original research articles published in peer-reviewed journals. Reference lists from retrieved studies as well as from recent reviews [19, 25-28] were also reviewed. 


\subsection{Inclusion and Exclusion Criteria}

To compare and quantify the effects of RSH vs. RSN in improving sea-level physical performance outcomes, the following inclusion criteria were considered: (1) single- or doubleblinded and placebo-controlled or cross-over design (i.e., with at least an intervention group completing RSN); (2) trained (i.e., regular training load $>2$ h.week ${ }^{-1}$ ) participants; (3) training intensity classified as 'all out', 'maximal' or 'supramaximal'; (4) sprint duration $\leq 30 \mathrm{~s}$, recovery duration $\leq 60 \mathrm{~s}$; (5) intervention duration $\geq 2$ weeks and (6) physical performance testing (laboratory or field; including at least repeated-sprint ability (RSA) or aerobic capacity from which $\mathrm{VO}_{2} \max$ could be determined) performed under normoxic conditions. Exclusion criteria were: (1) prior acclimatization/acclimation to hypoxia; (2) absence of physical performance measurement; (3) lack of a RSN group in the experimental design and/or (3) animal subjects.

\subsection{Data extraction}

A search of electronic databases and a scan of articles' reference list revealed 125 relevant studies (Fig. 1). Based on duplicates removal and screening of the title or abstract, 103 articles were dismissed. Twenty-two full-text articles were evaluated, and 9 were included for the metaanalysis. Each study was read and coded for descriptive variables: sex, training status, altitude level, intervention duration and frequency, training protocol.

Physical performance data were extracted in the forms of pre- (baseline) and post-training intervention (within 1-5 days; RSH vs. RSN) means, standard deviations (SDs), and sample sizes for RSH and RSN conditions. In studies that reported intermediate and post-intervention values, only post- values were recorded and compared with baseline. Data were collected directly from 
tables or within the text of the selected studies where possible or using Graph digitizing software (DigitizeIt, Germany) in studies where plots only were published. Dependent variables included best (i.e., fastest sprint time or highest power output [usually corresponding to the initial sprint] recorded/achieved during the RSA test) and mean (i.e., averaged sprint time or power output recorded/maintained throughout the test) RSA performances during repeated sprints. With the open-loop design, values were recalculated for an equal number of sprints performed by both groups, in order to allow comparison with the closed-loop design. Aerobic capacity was considered using direct (i.e., $\mathrm{VO}_{2}$ max or peak oxygen uptake $\left[\mathrm{VO}_{2}\right.$ peak]) or estimated (data were calculated from field test, e.g., distance covered during Yo-Yo intermittent recovery test level 1/2 or velocity at $\mathrm{VO}_{2} \max$ ) measurements of $\mathrm{VO}_{2} \max$.

\subsection{Data Analysis}

Meta-analysis was conducted using comprehensive meta-analysis software (version 2, Biostat, Inc., Englewood, NJ, USA) in order to aggregate, via a random-effects model [29], the standardized mean difference (SMD) between the effects of RSH vs. RSN on physical performance. Use of the SMD summary statistic allowed all effect sizes to be transformed into an uniform scale, which was then interpreted according to Cohen's conventional criteria [30] with SMDs of $<0.2,0.2-0.3,0.5$, and 0.8 representing trivial, small, medium, and large effect sizes, respectively. Heterogeneity was determined using the $I^{2}$ value, with values of 25,50 and 75 indicating low, moderate and high heterogeneity, respectively [29]. Study characteristics are presented as mean \pm SD unless otherwise stated. Potential publication bias was evaluated using 
Begg and Mazumdar's rank correlation and Egger's regression tests [31], with asymmetry examination of funnel plots. A P value $\leq 0.05$ was considered statistically significant.

\section{Results}

\subsection{Study Characteristics and Publication Bias}

Participants along with training characteristics for the meta-analyzed studies are displayed in Table 1. A total of 7 studies comprised only male [9, 32-37], one study included only female [38], and another one recruited both sexes [39]. The mean number of participants was $26 \pm 12$. Participants' age, height and body weight were $22.6 \pm 6.1$ years, $175.8 \pm 7.6 \mathrm{~cm}$ and $71.3 \pm 10.8$ $\mathrm{kg}$, respectively.

The mode of exercise primarily involved running (four studies; overground and/or treadmill runs; [34-37]) and cycling (four studies; ergocycle [9, 33, 37, 38]); and one study used double-poling [39]. Training intervention average duration was $3.7 \pm 1.3$ weeks with $2.6 \pm 0.6$ sessions per week. Exercise protocol consisted of $3 \pm 1$ sets, $7 \pm 4$ repetitions, $8 \pm 2 \mathrm{~s}$ of effort duration with $27 \pm 8 \mathrm{~s}$ of recovery and $7 \pm 5 \mathrm{~min}$ of inter-set rest.

Regarding testing, four different exercise modes (i.e., overground and treadmill running, cycling, double-poling) were used. These RSA protocols also differed in terms of number of sprint repetitions (i.e., from 6 to 10 repetitions for closed-loop design), duration/length of efforts (i.e., 7$10 \mathrm{~s}$ or $20-30 \mathrm{~m}$ ) as well as recovery time (i.e., 20-30 s) and type (i.e., passive or active). Similarly, aerobic capacity was assessed using either direct (from expired gas during laboratorybased incremental protocols) or estimated (from distance covered during field-based high- 
intensity intermittent protocols or velocity at $\mathrm{VO}_{2} \mathrm{max}$ during field incremental protocols) measurements of $\mathrm{VO}_{2} \max$.

Visual examination of the funnel plots (not presented), Begg and Mazumdar's rank correlation test $(\mathrm{P} \geq 0.11)$ and the Egger's regression test $(\mathrm{P} \geq 0.36)$ did not indicate the presence of potential publication bias for the SMDs in best and mean performance during repeated sprinting and $\mathrm{VO}_{2} \mathrm{max}$ in the studies included in the meta-analysis.

\subsection{Meta-analysis}

The forest plots depicting the individual SMD and associated $95 \%$ confidence interval (CI) and random-effects model for RSA best performance, mean RSA performance and $\mathrm{VO}_{2}$ max are shown in Figs. 2, 3 and 4, respectively.

Following data pooling, the SMD for mean RSA outcome was 0.46 (95\% CI $-0.02,0.93$ ), providing a significant small to moderate effect $(\mathrm{P}=0.05)$ in favor of RSH $v s . \mathrm{RSN}$, as shown in Fig. 3. Likewise, the effect on best RSA performance was higher with RSH compared with RSN $(\mathrm{SMD}=0.31,95 \% \mathrm{CI}-0.27,0.89$; small to moderate effect; $\mathrm{P}=0.30)$ (Fig. 2). In addition, there was a trivial non-significant effect of $\mathrm{RSH} v s$. $\mathrm{RSN}$ on $\mathrm{VO}_{2} \max$ improvement $(\mathrm{SMD}=0.18,95 \%$ CI - $0.25,0.61 ; \mathrm{P}=0.41)$ (Fig. 4). Heterogeneity was not detected among studies assessing best $\left(I^{2}\right.$ $=11.14 \%)$ and mean RSA outcomes $\left(I^{2}=6.19 \%\right)$ or $\mathrm{VO}_{2} \max \left(I^{2}=0.00 \%\right)$.

\section{Discussion}


The aggregated findings indicate that RSH is more effective than RSN for improving best (SMD $=0.31$, small to moderate beneficial effect) and mean $(\mathrm{SMD}=0.46$, small to moderate beneficial effect) RSA outcomes, as well as $\mathrm{VO}_{2} \max (\mathrm{SMD}=0.18$, trivial beneficial effect).

Irrespective of the repeated-sprint training components (i.e., exercise modality and exercise:recovery ratio) or participants' background, the results of this meta-analysis confirm the respective conclusions of the majority of both best and mean RSA studies ( 6 out of 9 studies for best RSA performance; 8 out of 9 studies for mean RSA performance) which were that RSH has a small to moderately greater beneficial effect than RSN on RSA outcomes (Figs. 2 and 3). Faiss et al. $[9,39]$ first showed that RSH delays task failure during a RSA test to exhaustion in trained cyclists and elite cross-country skiers (i.e., $+40 \%$ and $+58 \%$ for the number of sprints performed post RSH vs. RSN). Their results also showed that RSH was as efficient as RSN for improving power output on a single sprint (5-7\%) but with fatigue resistance being improved during sealevel repeated sprinting post-RSH only [9]. In the present systematic review, we recalculated from their results the peak ( +4 and $+5 \%$ relative to $\mathrm{RSN})$ and mean $(+5 \%$ and $+12 \%$ relative to RSN) power outputs at the same number of sprints performed for pre-post comparisons of the effects of RSH and RSN (i.e., $9^{\text {th }}$ sprint in Faiss et al. [9] and $11^{\text {th }}$ sprint in Faiss et al. [39]). This approach further pinpoints the putative benefit of RSH relative to RSN and allows comparison with other RSH studies. Because different RSA outcomes (i.e., peak, mean power outputs or best time; mean or total time; sprint decrement, fatigue index) were used in the different RSH studies, this meta-analysis reinforces these findings as, from all included studies, we carefully reported best and mean RSA performance across an equivalent number of sprints performed for both RSH and RSN groups. 
Our understanding of the physiological adaptations mediating physical performance enhancement in response to normoxic RSA is growing [25-27]. However, research about the underpinning mechanisms associated with the novel RSH method is still in its infancy. A solid ground suggests that RSH mechanisms likely differ from those associated to 'intermittent hypoxic training' [1618]. With maximal intensity efforts performed in hypoxia, an enhanced oxygen utilization (via improved blood perfusion level) and an improved behavior of fast-twitch fibers are expected compared with similar training at sea-level $[9,39]$. Pending confirmatory research, this could be based on at least three mechanisms: firstly, the compensatory vasodilatation with an induced nitric oxide (NO)-dependent increase in muscle blood flow aiming to match the increased oxygen demand at the muscular level when exercising in hypoxia [40, 41]; secondly, a greater microvascular oxygen delivery to fast-twitch fibers [42] mainly due to their higher fractional oxygen extraction [43]; thirdly, specific molecular adaptations arising from the oxygen-sensing pathway [15-18]. In support, previous animal model studies have highlighted phenotypic changes in favor of fast-twitch glycolytic fibers after hypoxia but not normoxia $[10,11]$. Furthermore, and despite reporting no additive effect on performance, Montero \& Lundby [44] demonstrated a marked RSH-induced increase in skeletal muscle concentration of total hemoglobin/myoglobin (considered an index of blood perfusion) compared with RSN and therefore confirmed similar findings on muscle oxygenation [9, 39]. While peak muscle perfusion is not reached with RSN [45], RSH should be associated with elevated muscle blood flow and eventually an increased endothelial shear stress, which in turn may stimulate angiogenesis in skeletal muscle [46, 47]. One cannot rule out that other potential mechanisms may be at play: it is known that, at the muscular level, waste metabolites accumulation and energy supply are essential limiting factors for RSA [25]. During repeated sprints, phosphocreatine breakdown is very high [48] and inorganic phosphate (Pi) accumulates in muscle. Since increased Pi levels may participate in 
decreasing the ability for force production, especially in fast-twitch fibers recruited during such fatiguing exercise [49], improved waste metabolites removal when blood flow is raised [50] (as reported post-RSH [9]) might delay fatigue during a RSA test.

A trivial beneficial effect of RSH on aerobic capacity (Fig. 4) compared with RSN was noted (5 out of 8 studies in RSH vs. RSN). Although calculations were based on the results of different field-based tests and not systematically from directly measured $\mathrm{VO}_{2} \max$ values, this observation remains practically relevant. However, this variable may not always reflect improvement in the sport-specific aerobic component. While Brocherie et al. [35] failed to show any additional RSHrelated effect on velocity at $\mathrm{VO}_{2}$ max using a modified version of the University of Montreal Track Test (i.e., the VAMEVAL maximal incremental running test) [51], sport-specific aerobic tests such as Yo-Yo intermittent recovery tests [52] may be more appropriate. Reportedly, a 4weeks treadmill RSH induced a $+33 \%$ improvement in Yo-Yo intermittent recovery test level 1 compared with RSN (+14\%) [36]. This would indicate that RSH may induce higher muscular oxidative activity rather than non-oxidative metabolism compared with RSN. Furthermore, a combination of methods improving RSA (using $\mathrm{RSH}$ ) and $\mathrm{VO}_{2} \max$ (via hemoglobin mass improvement through 'live high-train low' training camps) could optimize the benefits of acute and prolonged hypoxic stress [34] as proposed earlier [3].

Although the heterogeneity of the outcomes was low, a potential limitation of this meta-analysis concerns the different training and testing protocols used among the analyzed studies. The duration of RSH interventions ranged 2-5 weeks with 2-3 sessions per week. Further, training protocols considerably differed with 1-5 sets, 4-10 repetitions, 5-10 s efforts, 20-45 s recovery and 4.5-10 min inter-set duration, which may account for inconsistent findings. This may have impacted the physiological adaptations and/or physical performance, influenced by the various 
tests used (e.g., four different modes of testing [overground and treadmill running, cycling, double-poling] and the wide ranging exercise:recovery ratio), in the absence of a 'gold standard' for RSA test. Additionally, apart from the study by Brocherie et al. [34], the current literature has not yet investigated the delayed effects (i.e., after few weeks) of RSH interventions.

\section{Protocol recommendations}

Bearing in mind that effectiveness and adherence to RSH protocols are undoubtedly specific and individual, suggested recommendations are provided in Table 2. With a protocol resembling the actual recommendations, Brocherie et al. [53] demonstrated that RSH appears sufficient in severity, duration and/or frequency to elicit a significant hypoxic 'acclimation', with psychophysiological responses (i.e., overall peripheral discomfort, difficulty breathing and lower-limb discomfort) not negatively altered in comparison with RSN. With the great variation in exercise:recovery ratio among the RSH protocols, the recommendations made in Table 2 regarding exercise and recovery duration, as well as number of sets and repetitions, do not preclude longer duration (e.g., $\geq 30 \mathrm{~s}$ recovery) $\mathrm{RSH}$ protocols (including different number of sets and repetitions) being potentially more appropriate for specific physiological (e.g., oxidative vs. glycolytic component) and physical development (e.g., $\mathrm{VO}_{2} \mathrm{max}$ ). Regarding recovery type, applying active recovery (i.e., at low-to-moderate intensity) under hypoxic conditions may not be the most efficient. Hence, it may alter performance of subsequent sprint efforts, in particular via a slowing down of muscle re-oxygenation rate [9] and could lead to premature fatigue. In support, a previous study [54] conducted in normoxia indicated that active recovery (i.e., $50 \%$ of velocity at $\mathrm{VO}_{2} \mathrm{max}$ ) induced a lower replenishment of oxygen in myoglobin and hemoglobin and a 
reduced rate of phosphocreatine resynthesis from the previous intense effort. Furthermore, exercise mode (e.g., cycling or running) selection may also impact the magnitude of sportspecific fitness improvements. On the one hand, the non-weight-bearing nature of stationary cycling, coupled with minimal eccentric contraction of leg muscles, seems to mitigate risk of injury and discomfort [28]. On the other hand, neuromuscular fatigue is higher in cycling- vs. running-RSA modes [55]. Taking these factors into consideration, well-designed protocols using sport-specific conditions and/or ecological settings [34, 56, 57] would allow more effective application of research findings in field conditions. Improved understanding of operative mechanisms is also still needed. We also acknowledge that other variations of RSH could be even more effective for specific sports or playing position. Therefore, our recommendations should be seen as a starting point and we encourage practitioners to challenge and refine them as appropriate.

\section{Perspectives}

Given the small number of RSH studies conducted to date, there are still important questions that need to be addressed. These include the question of the optimal combination of variables such as sprint length (m)/duration (s), exercise:recovery ratio (from 1:2 through 1:5), inter-set recovery duration, number of sets and/or repetitions, and/or session frequency and their effects on physiological adaptations and related physical improvements, whether 'anaerobic' and/or 'aerobic'. This may also provide valuable insights in terms of participants' adherence to training, delayed onset muscle soreness and injury occurrence, and may allow specific prescription guidelines to be recommended. In this view, a particular focus on elite intermittent-sport athletes 
may be helpful. Furthermore, as RSH is generally combined with other sea-level conditioning training (e.g., resistance, aerobic), determining the optimal arrangement of these different types of training is also warranted because the interaction between several approaches is unknown. Finally, to deepen our understanding of the physiological mechanisms underlying RSH, comparisons of different hypoxic stresses (i.e., normobaric [all studies were conducted in this environment] $v s$. hypobaric hypoxia) and different environmental stresses (i.e., hypoxic vs. heat vs. control) need to be conducted.

\section{Conclusion}

The current meta-analysis provides evidence that RSH is an effective training strategy for improving sport-specific physical performance among athletes and induces greater gains in RSA than RSN. Indeed, RSH induces small to moderately greater best and mean RSA performance improvements than RSN among endurance and team-sport athletes. The additional benefit observed for $\mathrm{VO}_{2}$ max was trivially higher for RSH vs. RSN.

\section{Compliance with Ethical Standards}

Funding

No sources of funding were used to assist in the preparation of this article.

Conflicts of interest 
Franck Brocherie, Olivier Girard, Raphaël Faiss and Grégoire P. Millet declare that they have no conflicts of interest relevant to the content of this review.

\section{References}

1. Hopkins WG, Hawley JA, Burke LM. Design and analysis of research on sport performance enhancement. Med Sci Sports Exerc. 1999 Mar;31(3):472-85.

2. Wilber RL. Application of altitude/hypoxic training by elite athletes. Med Sci Sports Exerc. 2007 Sep;39(9):1610-24.

3. Millet GP, Roels B, Schmitt L, et al. Combining hypoxic methods for peak performance. Sports Med. 2010 Jan 1;40(1):1-25.

4. Levine BD, Stray-Gundersen J. "Living high-training low": effect of moderate-altitude acclimatization with low-altitude training on performance. J Appl Physiol. 1997 Jul;83(1):10212.

5. Millet GP, Faiss R, Brocherie F, et al. Hypoxic training and team sports: a challenge to traditional methods? Br J Sports Med. 2013 Dec;47 Suppl 1:i6-i7.

6. Girard O, Amann M, Aughey R, et al. Position statement--altitude training for improving team-sport players' performance: current knowledge and unresolved issues. Br J Sports Med. 2013 Dec;47 Suppl 1:i8-16.

7. Girard O, Brocherie F, Millet GP. On the use of mobile inflatable hypoxic marquees for sport-specific altitude training in team sports. Br J Sports Med. 2013 Dec;47 Suppl 1:i121-i3.

8. Faiss R, Girard O, Millet GP. Advancing hypoxic training in team sports: from intermittent hypoxic training to repeated sprint training in hypoxia. Br J Sports Med. 2013 Dec;47 Suppl 1:i45-i50.

9. Faiss R, Leger B, Vesin JM, et al. Significant molecular and systemic adaptations after repeated sprint training in hypoxia. PLoS One. 2013;8(2):e56522.

10. Kawada S, Ishii N. Changes in skeletal muscle size, fibre-type composition and capillary supply after chronic venous occlusion in rats. Acta Physiol (Oxf). 2008 Apr;192(4):541-9.

11. Ishihara $\mathrm{A}$, Itho $\mathrm{K}$, Itoh $\mathrm{M}$, et al. Hypobaric-hypoxic exposure and histochemical responses of soleus muscle fibers in the rat. Acta Histochem. 1994 Mar;96(1):74-80.

12. Mounier R, Pedersen BK, Plomgaard P. Muscle-specific expression of hypoxia-inducible factor in human skeletal muscle. Exp Physiol. 2010 Aug;95(8):899-907.

13. Levine BD, Stray-Gundersen J. Dose-response of altitude training: how much altitude is enough? Adv Exp Med Biol. 2006;588:233-47.

14. Wilber RL, Stray-Gundersen J, Levine BD. Effect of hypoxic "dose" on physiological responses and sea-level performance. Med Sci Sports Exerc. 2007 Sep;39(9):1590-9.

15. Semenza GL, Shimoda LA, Prabhakar NR. Regulation of gene expression by HIF-1. Novartis Found Symp. 2006;272:2-8; discussion -14, 33-6.

16. Calbet JA, Lundby C. Air to muscle $\mathrm{O} 2$ delivery during exercise at altitude. High Alt Med Biol. 2009 Summer;10(2):123-34.

17. Hoppeler H, Vogt M. Muscle tissue adaptations to hypoxia. J Exp Biol. $2001 \mathrm{Sep} ; 204(\mathrm{Pt}$ 18):3133-9. 
18. Lundby C, Calbet JA, Robach P. The response of human skeletal muscle tissue to hypoxia. Cell Mol Life Sci. 2009 Nov;66(22):3615-23.

19. McLean BD, Gore CJ, Kemp J. Application of 'live low-train high' for enhancing normoxic exercise performance in team sport athletes. Sports Med. 2014 Sep;44(9):1275-87.

20. Lundby C, Robach P. Does 'altitude training' increase exercise performance in elite athletes? Exp Physiol. 2016 Jul 1;101(7):783-8.

21. Millet GP, Brocherie F, Faiss R, et al. Clarification on altitude training. Exp Physiol. 2016 Oct 17; [Epub ahead of print].

22. Montero D, Lundby C. Enhanced performance after repeated sprint training in hypoxia: false or reality? Med Sci Sports Exerc. 2015 Nov;47(11):2483.

23. Faiss R, Holmberg HC, Millet GP. Response. Med Sci Sports Exerc. 2015 Nov;47(11):2484.

24. Moher D, Liberati A, Tetzlaff J, et al. Preferred reporting items for systematic reviews and meta-analyses: the PRISMA statement. BMJ. 2009;339:b2535.

25. Girard O, Mendez-Villanueva A, Bishop D. Repeated-sprint ability - part I: factors contributing to fatigue. Sports Med. 2011 Aug 1;41(8):673-94.

26. Bishop D, Girard O, Mendez-Villanueva A. Repeated-sprint ability - part II: recommendations for training. Sports Med. 2011 Sep 1;41(9):741-56.

27. Taylor J, Macpherson T, Spears I, et al. The effects of repeated-sprint training on fieldbased fitness measures: a meta-analysis of controlled and non-controlled trials. Sports Med. 2015 Jun;45(6):881-91.

28. Gist NH, Fedewa MV, Dishman RK, et al. Sprint interval training effects on aerobic capacity: a systematic review and meta-analysis. Sports Med. 2014 Feb;44(2):269-79.

29. Higgins JP, Thompson SG, Deeks JJ, et al. Measuring inconsistency in meta-analyses. BMJ. 2003 Sep 6;327(7414):557-60.

30. Cohen J. Statistical power analysis for the behavioral sciences. Hillsdale, NJ: Lawrence Erlbaum Associates; 1988.

31. Egger M, Davey Smith G, Schneider M, et al. Bias in meta-analysis detected by a simple, graphical test. BMJ. 1997 Sep 13;315(7109):629-34.

32. Goods PS, Dawson B, Landers GJ, et al. No additional benefit of repeat-sprint training in hypoxia than in normoxia on sea-level repeat-sprint ability. J Sports Sci Med. 2015 Sep;14(3):681-8.

33. Montero D, Lundby C. Repeated sprint training in hypoxia versus normoxia does not improve performance: a double-blind and cross-over study. Int J Sports Physiol Perform. 2016 May 3;[Epub ahead of print].

34. Brocherie F, Millet GP, Hauser A, et al. "Live High-Train Low and High" hypoxic training improves team-sport performance. Med Sci Sports Exerc. 2015 Oct;47(10):2140-9.

35. Brocherie F, Girard O, Faiss R, et al. High-intensity intermittent training in hypoxia: a double-blinded, placebo-controlled field study in youth football players. J Strength Cond Res. 2015 Jan;29(1):226-37.

36. Galvin HM, Cooke K, Sumners DP, et al. Repeated sprint training in normobaric hypoxia. Br J Sports Med. 2013 Dec;47 Suppl 1:i74-i9.

37. Gatterer H, Philippe M, Menz V, et al. Shuttle-run sprint training in hypoxia for youth elite soccer players: a pilot study. J Sports Sci Med. 2014 Dec;13(4):731-5.

38. Kasai N, Mizuno S, Ishimoto S, et al. Effect of training in hypoxia on repeated sprint performance in female athletes. Springerplus. 2015;4:310. 
39. Faiss R, Willis S, Born DP, et al. Repeated double-poling sprint training in hypoxia by competitive cross-country skiers. Med Sci Sports Exerc. 2015 Apr;47(4):809-17.

40. Casey DP, Joyner MJ. Compensatory vasodilatation during hypoxic exercise: mechanisms responsible for matching oxygen supply to demand. J Physiol. 2012 Dec 15;590(Pt 24):6321-6.

41. Gonzalez-Alonso J, Mortensen SP, Dawson EA, et al. Erythrocytes and the regulation of human skeletal muscle blood flow and oxygen delivery: role of erythrocyte count and oxygenation state of haemoglobin. J Physiol. 2006 Apr 1;572(Pt 1):295-305.

42. Cleland SM, Murias JM, Kowalchuk JM, et al. Effects of prior heavy-intensity exercise on oxygen uptake and muscle deoxygenation kinetics of a subsequent heavy-intensity cycling and knee-extension exercise. Appl Physiol Nutr Metab. 2012 Feb;37(1):138-48.

43. McDonough P, Behnke BJ, Padilla DJ, et al. Control of microvascular oxygen pressures in rat muscles comprised of different fibre types. J Physiol. 2005 Mar 15;563(Pt 3):903-13.

44. Montero D, Lundby C. Repeated Sprint Training in Hypoxia Versus Normoxia Does Not Improve Performance: A Double-Blind and Cross-Over Study. Int J Sports Physiol Perform. 2016 May 3.

45. Calbet JA, Lundby C. Skeletal muscle vasodilatation during maximal exercise in health and disease. J Physiol. 2012 Dec 15;590(24):6285-96.

46. Hellsten Y, Hoier B. Capillary growth in human skeletal muscle: physiological factors and the balance between pro-angiogenic and angiostatic factors. Biochem Soc Trans. 2014 Dec;42(6):1616-22.

47. Ridnour LA, Isenberg JS, Espey MG, et al. Nitric oxide regulates angiogenesis through a functional switch involving thrombospondin-1. Proc Natl Acad Sci U S A. 2005 Sep 13;102(37):13147-52.

48. Bogdanis GC, Nevill ME, Boobis LH, et al. Contribution of phosphocreatine and aerobic metabolism to energy supply during repeated sprint exercise. J Appl Physiol. 1996 Mar;80(3):876-84.

49. Westerblad H, Allen DG, Lannergren J. Muscle fatigue: lactic acid or inorganic phosphate the major cause? News Physiol Sci. 2002 Feb;17:17-21.

50. Endo M, Okada Y, Rossiter HB, et al. Kinetics of pulmonary VO2 and femoral artery blood flow and their relationship during repeated bouts of heavy exercise. Eur J Appl Physiol. 2005 Dec;95(5-6):418-30.

51. Leger LA, Boucher R. An indirect continuous running multistage field test: the Universite de Montreal track test. Can J Appl Sport Sci. 1980 Jun;5(2):77-84.

52. Bangsbo J, Iaia FM, Krustrup P. The Yo-Yo intermittent recovery test: a useful tool for evaluation of physical performance in intermittent sports. Sports Med. 2008;38(1):37-51.

53. Brocherie F, Millet GP, Girard O. Psycho-physiological responses to repeated-sprint training in normobaric hypoxia and normoxia. Int J Sports Physiol Perform. 2016 May 3;[Epub ahead of print].

54. Dupont G, Blondel N, Berthoin S. Performance for short intermittent runs: active recovery vs. passive recovery. Eur J Appl Physiol. 2003 Aug;89(6):548-54.

55. Rampinini E, Connolly DR, Ferioli D, et al. Peripheral neuromuscular fatigue induced by repeated-sprint exercise: cycling vs. running. J Sports Med Phys Fitness. 2016 Jan-Feb;56(12):49-59.

56. Girard O, Racinais S, Kelly L, et al. Repeated sprinting on natural grass impairs vertical stiffness but does not alter plantar loading in soccer players. Eur J Appl Physiol. 2011 Oct;111(10):2547-55. 
57. Brocherie F, Millet GP, Girard O. Neuro-mechanical and metabolic adjustments to the repeated anaerobic sprint test in professional football players. Eur J Appl Physiol. 2015 May;115(5):891-903. 


\section{Figures legend}

Fig. 1. Flow chart of study selection.

Fig. 2. Forest plot of the standardized mean difference (SMD) between the effect of repeatedsprint training performed in hypoxia (RSH) vs. normoxia (RSN) and/or control on best RSA performance. Squares represent the SMD for each study. The diamond represents the pooled SMD for all studies. 95\% CI, 95\% confidence interval; $d f$, degrees of freedom; $I V$, inverse variance. Std, standardized; RSA, repeated-sprint ability.

Fig. 3. Forest plot of the standardized mean difference (SMD) between the effect of repeatedsprint training performed in hypoxia (RSH) $v s$. normoxia (RSN) and/or control on mean RSA performance. Squares represent the SMD for each study. The diamond represents the pooled SMD for all studies. $95 \% C I, 95 \%$ confidence interval; $d f$, degrees of freedom; $I V$, inverse variance. Std, standardized; RSA, repeated-sprint ability.

Fig. 4. Forest plot of the standardized mean difference (SMD) between the effect of repeatedsprint training performed in hypoxia (RSH) vs. normoxia (RSN) and/or control on maximal oxygen uptake $\left(\mathrm{VO}_{2} \mathrm{max}\right)$. Squares represent the SMD for each study. The diamond represents the pooled SMD for all studies. $95 \% C I, 95 \%$ confidence interval; $d f$, degrees of freedom; $I V$, inverse variance. Std, standardized; RSA, repeated-sprint ability. 


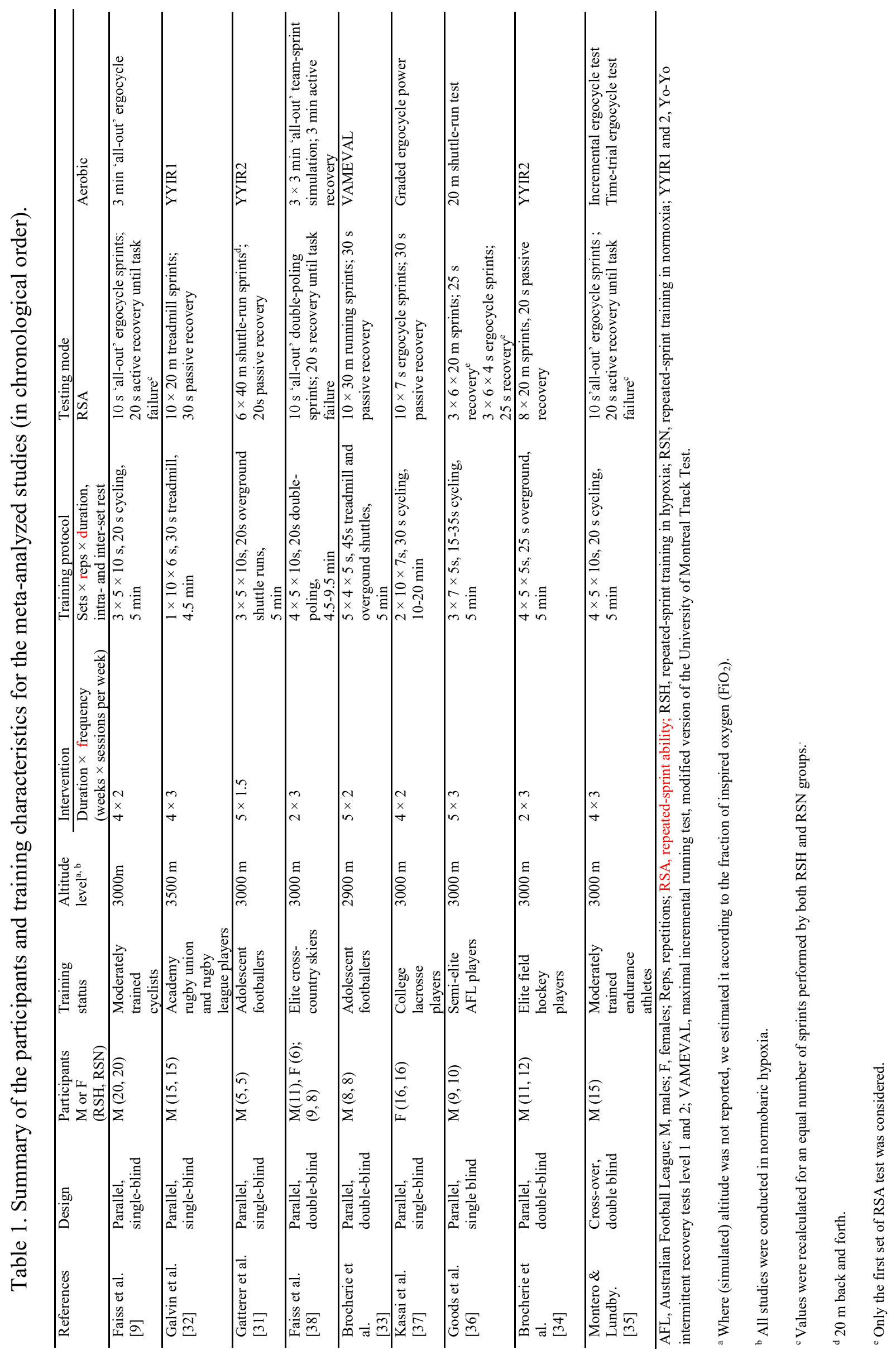


Table 2. Protocol recommendations for repeated-sprint training in hypoxia (RSH).

\begin{tabular}{|c|c|}
\hline Frequency & 2-3 sessions per week. \\
\hline Periodization & Blocks of 2-5 weeks. \\
\hline Duration & $\sim 60$ min (including warm-up and cool-down). \\
\hline Modality & $\begin{array}{l}\text { Sport-specific (overground/treadmill, ergocycle, double-poling } \\
\text { ergometer, etc.). }\end{array}$ \\
\hline Intensity & $\begin{array}{l}\text { Exercise = maximal, supra-maximal, 'all-out' efforts. } \\
\text { Inter-sprints recovery = passive. } \\
\text { Inter-sets rest = passive. }\end{array}$ \\
\hline Interval times & $\begin{array}{l}\text { Exercise }=3-4 \text { sets of } 4-7 \times 4-15 \text {-s intervals. } \\
\text { Inter-sprints recovery } \leq 30 \mathrm{~s} \text {. } \\
\text { Exercise:recovery ratio }=1: 2 \text { to } 1: 5 \\
\text { Inter-sets rest }=3-5 \mathrm{~min} .\end{array}$ \\
\hline
\end{tabular}




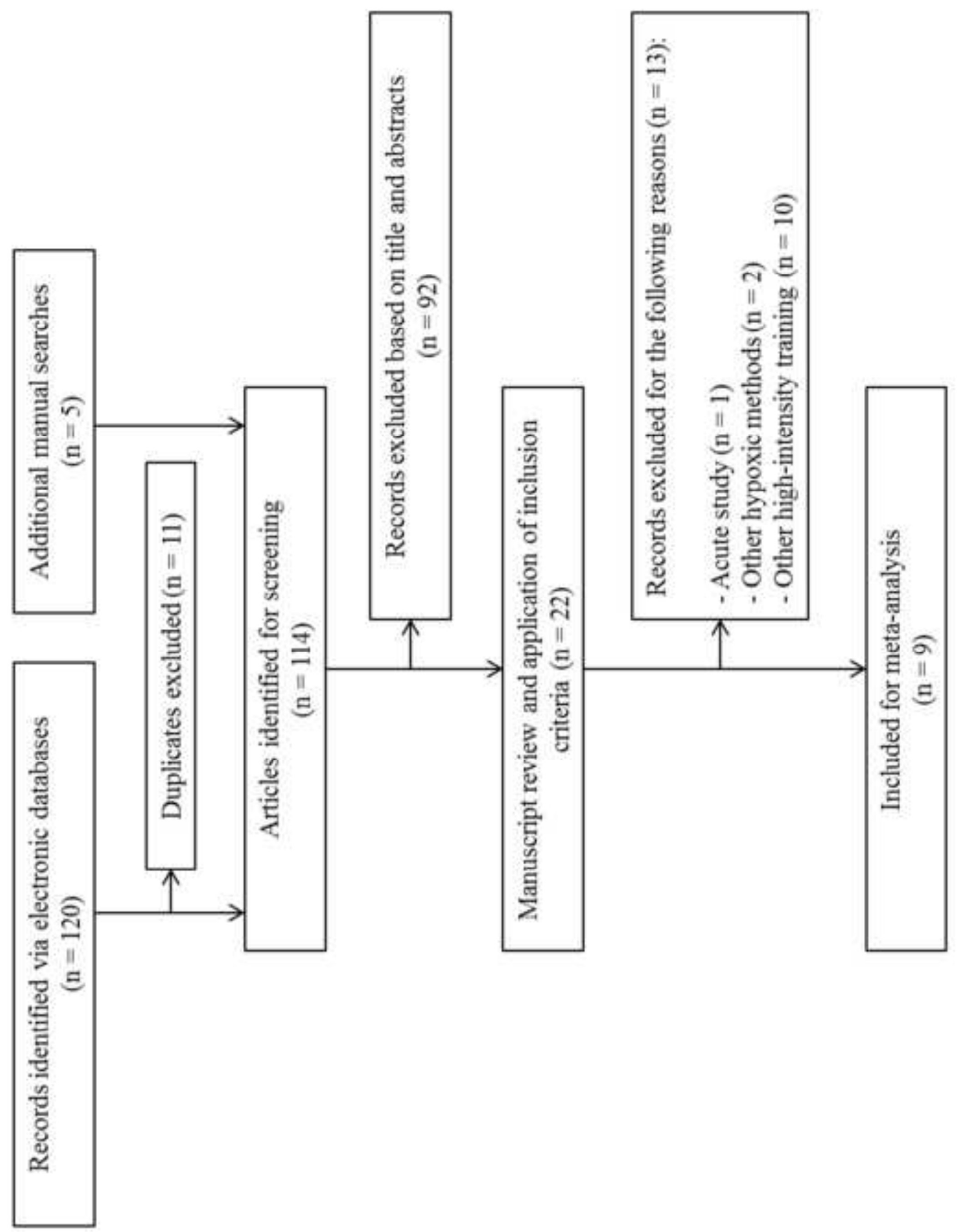



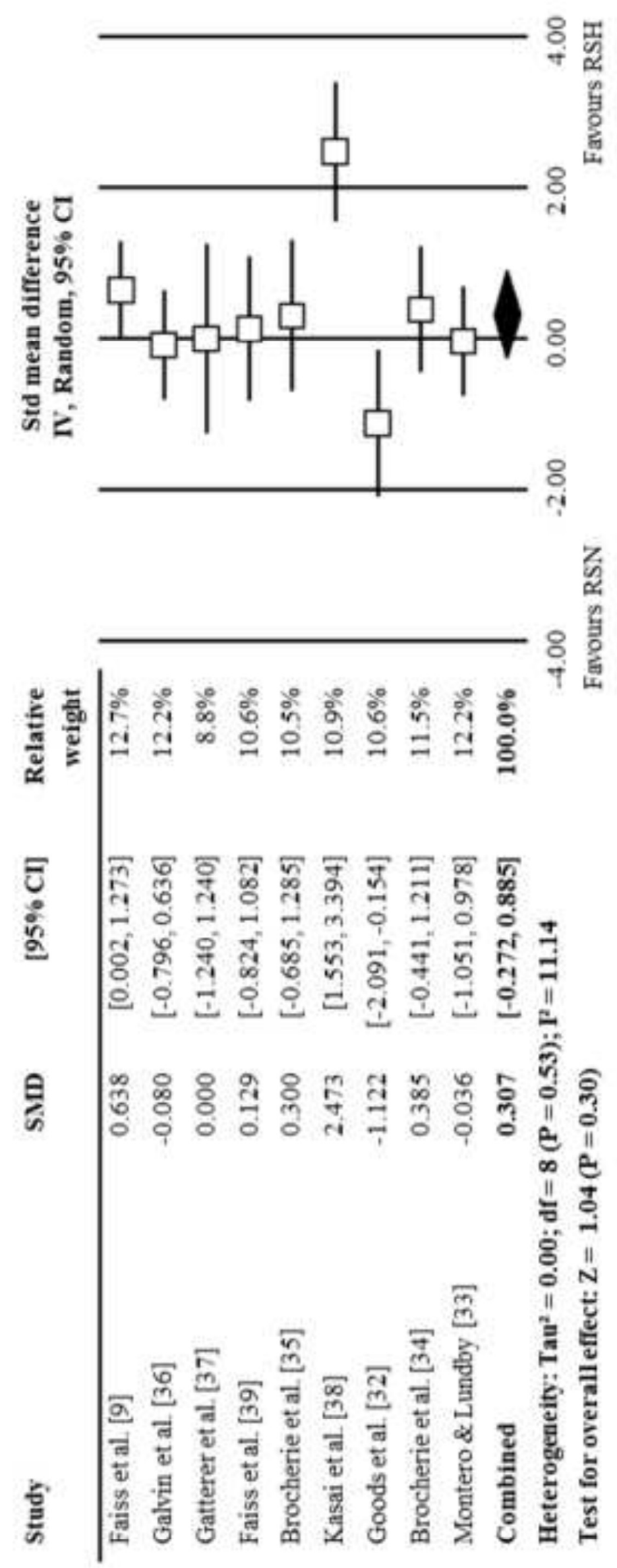

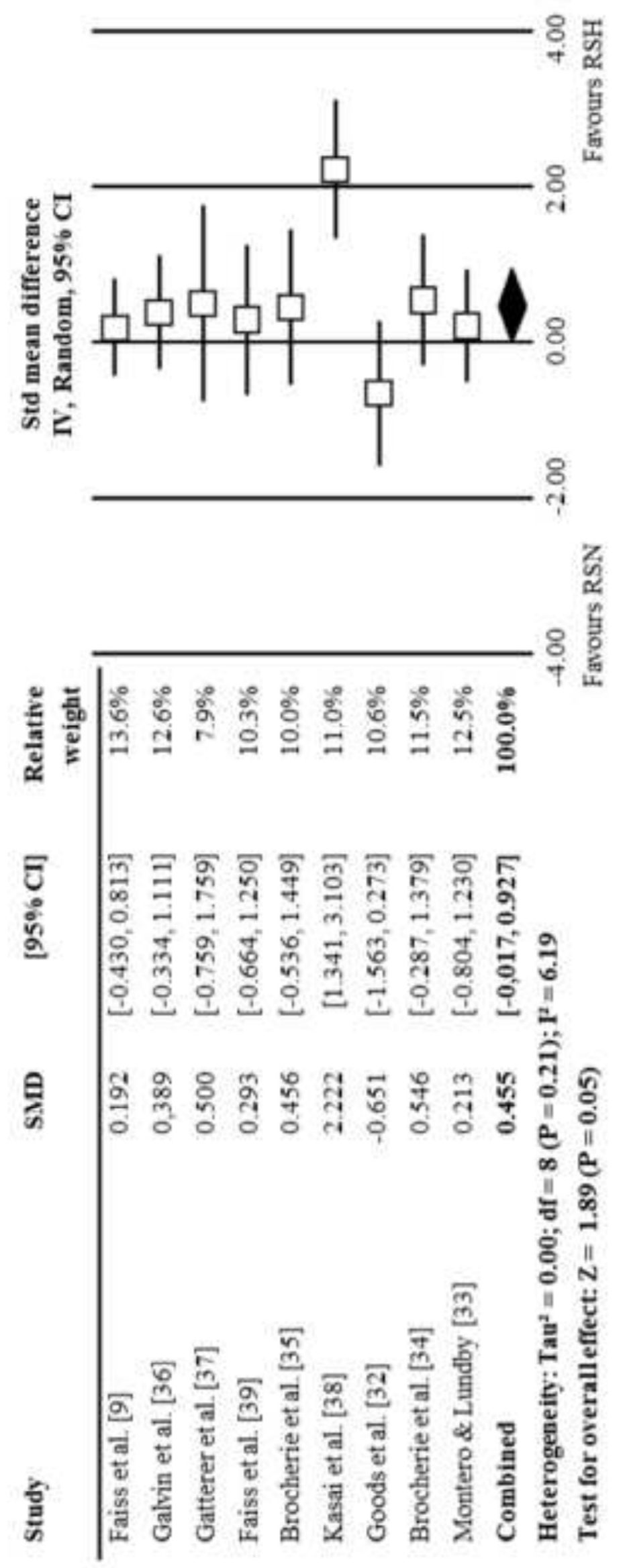